\title{
Compact Isoflux X-band Payload Telemetry Antenna with Simultaneous Dual Circular Polarization for LEO Satellite Applications
}

\author{
E. ARNAUD, J. DUGENET, K. ELIS, A. GIRARDOT, D. GUIHARD, C. MENUDIER, \\ T. MONEDIERE, F. ROZIERE, M. THEVENOT
}

\begin{abstract}
This paper describes a compact isoflux X-band payload telemetry and data handling antenna with simultaneous dual circular polarization for Low Earth Orbits (LEO) satellite applications. The main objective of this antenna is to use polarization diversity to improve the efficiency of the payload telemetry link. Some solutions have already been proposed to that end, but the studied antenna combines both good electromagnetic performances and compactness. This high power capability waveguide antenna presents an axial ratio (AR) lower than $2.5 \mathrm{~dB}$ over the 8.025-8.4 GHz frequency band at the limit of coverage, i.e. $65^{\circ}$, for any azimuth angle $(\varphi)$. It has been designed, realized and successfully measured.
\end{abstract}

Index Terms - Compact isoflux antenna, simultaneous dual circular polarization, high power capability

\section{INTRODUCTION}

$\mathrm{T}$ he studied antenna is intended to be mounted on a LEO microsatellite platform. To achieve its payload telemetry link, two families of antennas are usually considered: the first is based on isoflux pattern antennas and the second on high-gain directional antennas with beam steering. The use of directional antennas increases the data rate but requires a beam alignment by orienting the satellite or by equipping these antennas with beam steering devices, which makes this second solution expensive and unsuitable for most missions. Compared to beam steering solutions, the advantage of isoflux antennas is that they are low cost. The counterpart is the low level of accessible gain which constitutes a factor limiting the link speed. It is therefore relevant to look for new solutions, based on isoflux antennas, which would increase the speed of the payload telemetry link. Increasing the bandwidth of theses antennas and generating polarization diversity are two possible approaches. In this paper, the second solution was chosen. The antenna must then guarantee simultaneously a Right hand and Left Hand Circular Polarization (RHCP and LHCP) with a low Axial Ratio (AR) over the whole bandwidth and over a wide angular range. Moreover, the power handling capability of the proposed antenna must be quite high $(2 \mathrm{X} 50 \mathrm{~W})$. Over the last few years, many antennas have been identified as good

Manuscript received.

This work was cofunded by French Space Agency - CNES, XLIM and ArianeGroup

E. Arnaud, C. Menudier, T. Monediere and M. Thevenot are with the XLIM laboratory, Faculté des Sciences et Techniques, 123 avenue Albert Thomas, 87060 Limoges, France. (E-mail: eric.arnaud@xlim.fr). candidates to meet demands for either circular polarization, isoflux pattern or both criteria. The first one is the quadrifilar helix [1]-[2] which is capable of having an edge of coverage (EOC) angle up to $70^{\circ}$ but it often requires a considerable height and a complicated feeding system. The other most used antenna is the choke horn. This one has been employed for a long time for satellite communications. This kind of antenna was firstly used to feed a parabolic antenna with a focal length to diameter ratio (F/D) lower than 0.3 [3]. Geyer [4] and LaGrone [5] introduced a corrugated flange on the circular waveguide aperture in 1966. Wohlleben [6] and Shafai [7] have respectively improved in 1972 and 1977 this method in order to increase the radiating aperture angle. Brachat gives an approximate synthesis design process for this kind of antenna in 1994 [8]. He shows the need to reduce the amplitude of the feed aperture field at the center. Jeon and Kim use ridge structures, but it probably causes a deterioration of radiation pattern symmetry [9]-[10]. Ravanelli uses an open waveguide loaded with quartz and a series of slots across the corrugation walls [11]. Kilian use a small horn input diameter [12]. The antenna size of these last two designs is large. Unfortunately, an isoflux pattern with a high EOC angle $\left(>50^{\circ}\right)$ and a compact antenna are often incompatible. In [13], Caminita has found a solution to obtain good performances while having a reasonable antenna size feeding his antenna by a sub reflector system located at the center. On the other hand, the antenna weight was high.

In this study, a tradeoff between good electromagnetic performances, reasonable antenna dimensions, a symmetrical radiation pattern, high power handling, low sensitivity with respect to the platform and a light weight has been made to get the best design [14]. Consequently, this work relies on a choke horn, a conventional septum polarizer [15] and a dielectric cone with a particular shape at an open-ended waveguide to meet the desired antenna specifications provided by the French Spatial Agency (CNES) and presented in Table I. The optimal footprints for radiation patterns and AR are given in Fig. 1. In this contribution, a conical shape is used at the waveguide output, to help generate the isoflux radiation pattern, by drawing the energy from the vertical towards the horizontal

J. Dugenet, A. Girardot, D. Guihard and F. Rozière are with Ariane group 33166 St Médard en Jalles - France

K. Elis is with French Space Agency - C.N.E.S - 18 Avenue Edouard Belin, 31400 Toulouse France. 
direction [16]. This paper is organized as follows. An antenna description is given in section II. Then, the design is presented with two different dielectrics for the cone. The first one allows reaching the performance in EOC with no consideration of space environment. The second is proposed to comply with space constrains. A simulation of the first case and a comparison of the measured and theoretical results for the second case are presented in section III. Finally, a conclusion is given.

ANTENNA REQUIREMENTS

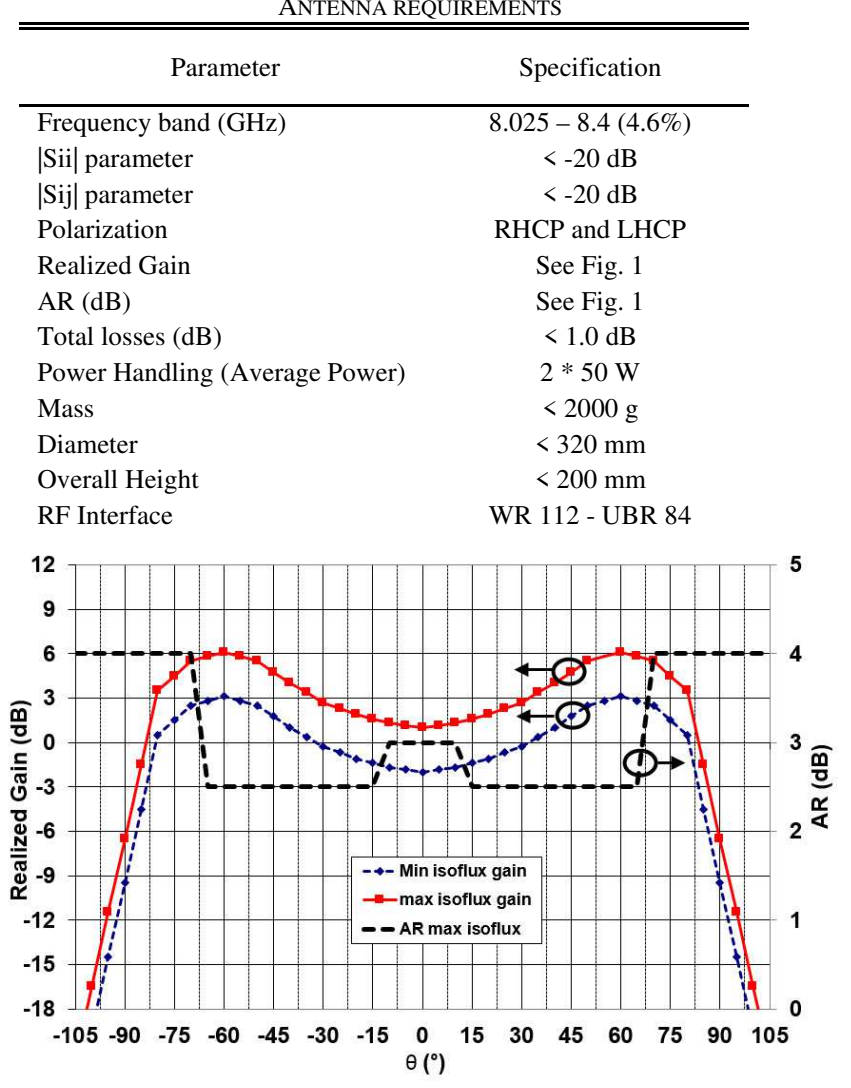

Fig. 1. Radiation pattern and AR objective footprints

\section{ANTENNA DESCRIPTION}

The detailed structure of the simulated antenna using CST Microwave Studio is presented in Fig. 2. In order to guarantee the power handling, a waveguide fed antenna has been chosen. It consists of four main parts:

- equal depth and width corrugated rings for the amplitude roll-off from antenna boresight to horizon and choke rings to have the peak gain at an EOC angle of $60^{\circ}$,

- a dielectric cone and circular waveguide $(\phi=24.7 \mathrm{~mm})$ to draw energy from the vertical direction towards the horizontal which reduces the antenna size (Fig. 3),

- a septum polarizer built in a square waveguide to have the desired AR and a good isolation between the ports,

- a quarter-wave impedance transformer and two waveguide bends to adapt half of the square waveguide of the polarizer to the WR 112 waveguide.

Notice that the higher the corrugated ring number, the greater the amplitude roll-off. There is a trade-off between the antenna size and this roll-off. The choice of the dielectric constant value is important: if too weak, then the cone has no electromagnetic effect, if too strong, then it is no longer possible to have a mechanically suitable cone.

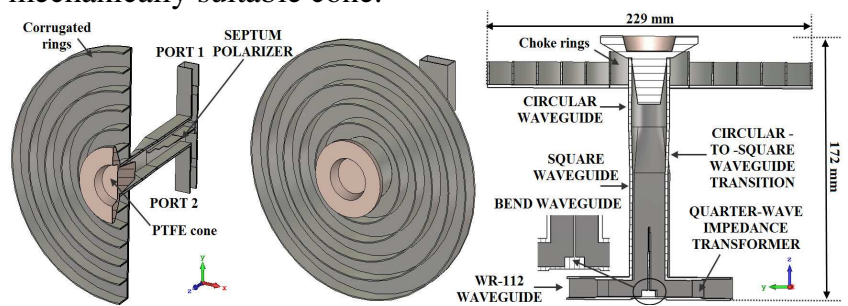

Fig. 2. Simulated antenna design

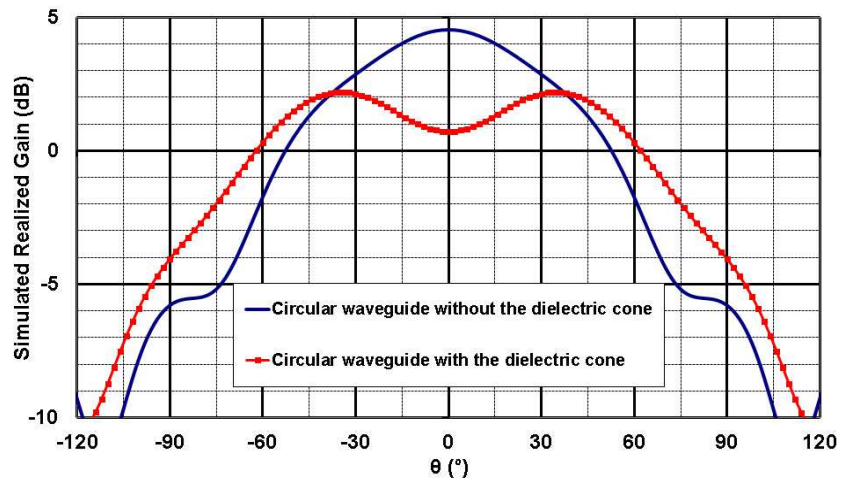

Fig. 3. Circular waveguide radiation pattern without or with the cone

This value must be between 2 and 4 . The bottom part of the cone has been optimized to minimize the discontinuity between air and dielectric, consequently minimizing the isolation between the ports. It has been gradually tapered back up to the circular waveguide diameter. The top part of the cone has been optimized to draw most of the energy towards the horizontal direction. In between, a part of the cone completely fills the circular waveguide. The advantage of this cone is that it is perfectly circular symmetric, which means that the radiation patterns are also symmetrical. Two cases have been studied. A first design has been made by taking a Polytetrafluoroethylene dielectric (P.T.F.E) and a second one by taking polyetheretherketone (P.E.E.K).

\section{MEASUREMENT AND SIMULATION}

\section{A. Simulation with a P.T.F.E cone $\left(\varepsilon_{r l}=2.1\right)$}

The design results of the antenna with the P.T.F.E cone are presented in Fig.4. Simulations of the stand-alone antenna were performed by XLIM. All simulated results meet the requirements of Table I, especially in terms of cross polarization discrimination $(\mathrm{XPD}<16.9 \mathrm{~dB}$, i.e. $\mathrm{AR}<2.5 \mathrm{~dB}$ up to $65^{\circ}$ ) and EOC angle (Fig. 4). Only the realized gain at $90^{\circ}$ is not achieved. In order to improve this parameter, other corrugated rings should be added. However, it is preferable to avoid using this type of material in a space environment. The new material must have a more stable and lower coefficient of thermal expansion than P.T.F.E in order to limit the impact of temperature deformations. The P.E.E.K GF30 (30\% glass fiber reinforced) has therefore been chosen for such conditions.

B. Simulation - Measurement with a P.E.E.K cone $\left(\varepsilon_{r 2}=3.7\right)$

Using a resonant cavity method at $4.5 \mathrm{GHz}$ and $10 \mathrm{GHz}$, and a reflection/reflection method in waveguide at $4,7 \mathrm{GHz}$, the 
average dielectric constant $\left(\varepsilon_{\mathrm{r} 2}\right)$ of the P.E.E.K is $3.7 \pm 0.04$ and its dissipation factor $(\tan \delta)$ is $0.004 \pm 0.0002$. A simple scaling of the cone by $\sqrt{\frac{\varepsilon_{r 1}}{\varepsilon_{r 2}}}$ is not possible to completely design the antenna based on the previous one.

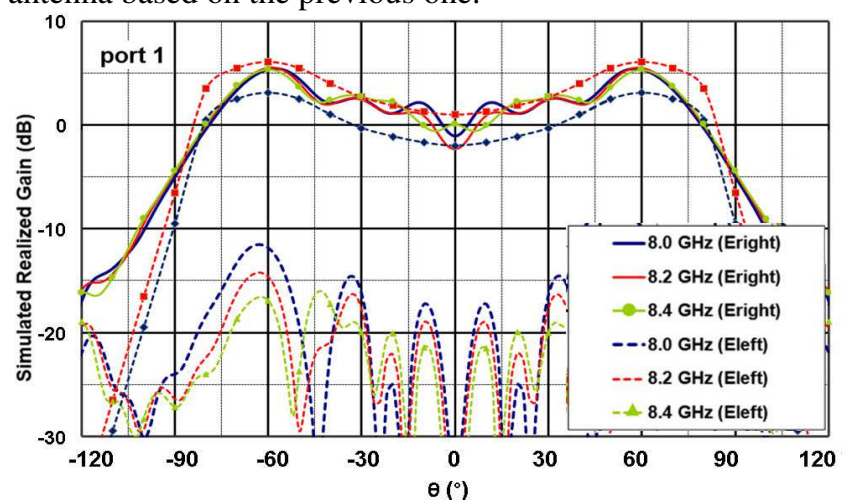

Fig. 4. Simulated radiation pattern with the P.T.F.E cone $\left(\varphi=0^{\circ}\right)$

The circular waveguide diameter must allow the wave propagation over the entire frequency band. Its diameter must be similar or close to the previous case. In addition, in order to have a mechanically suitable cone, this parameter is increased to $27.9 \mathrm{~mm}$. Six holes have been drilled to reduce the dielectric constant on this part and its shape has been slightly modified (Fig. 5). The cone has been fixed by three M3 peek screws. It was therefore necessary to increase the thickness of the central waveguide to $5 \mathrm{~mm}$. The thickness of the other rings and the septum is $1 \mathrm{~mm}$. Finally, it is compact (238 X $185 \mathrm{~mm})$. It is difficult to reduce this height significantly without degrading antenna performances. The polarizer height guarantees a good AR. The cone height contributes both to the radiation pattern and to the good isolation. The lengths of the circular waveguide and the circular-to-square waveguide transition are necessary to get a good isolation.

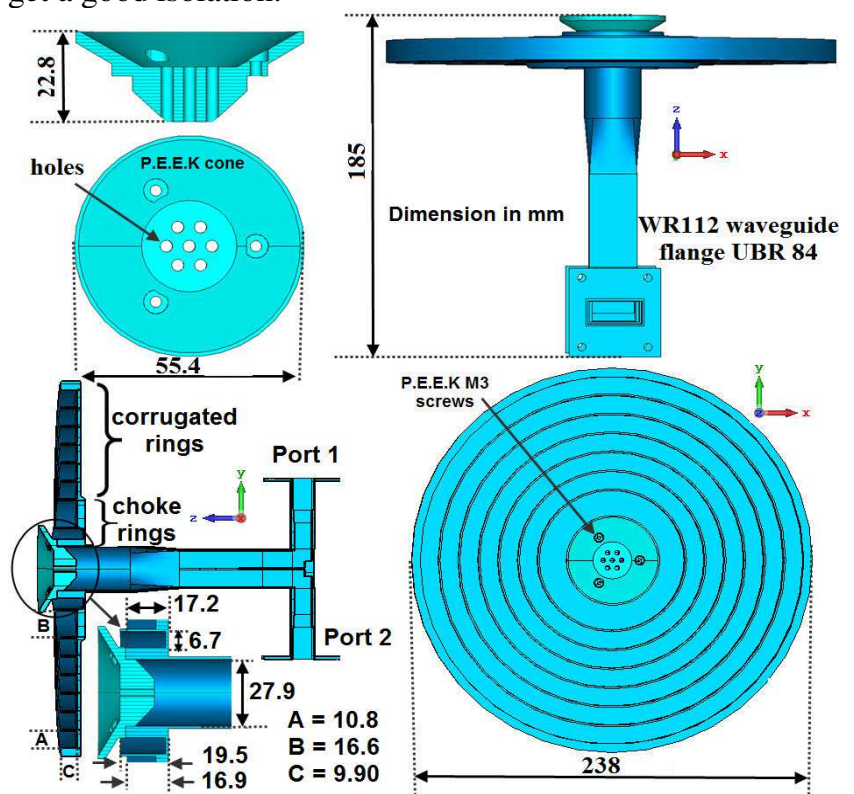

Fig. 5. Realized antenna design

The antenna was manufactured by ArianeGroup with a 3D metal printing process using Additive Layer Manufacturing (ALM), based on Electron Beam Melting (EBM) technology. The used material is titanium Ta6V $\left(5.910^{5} \mathrm{~S} / \mathrm{m}\right.$ of electric conductivity), allowing a low weight antenna (920 g). Fig. 6 shows the realized antenna photography before and after sandblasting, machining and electro-erosion of critical areas inside the waveguide. The simulated total efficiency of antenna is higher than $91 \%$ ( $<0.4 \mathrm{~dB}$ of total losses).

1) $\mathrm{S}_{\mathrm{ii}}$ and $\mathrm{S}_{\mathrm{ji}}$ parameters

Fig. 7 shows a comparison between the simulated and measured results of the [S] parameters. In both cases, the curves show the $[\mathrm{S}]$ parameters lower than the expected "-20dB" value on the whole bandwidth even if a difference appears between simulation and measurement.

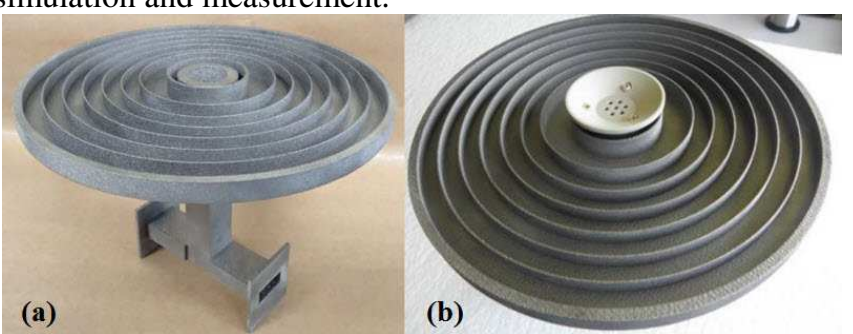

Fig. 6. Manufactured antenna photography: (a) before the mechanical treatment (b) after the mechanical treatment and cone positioning.

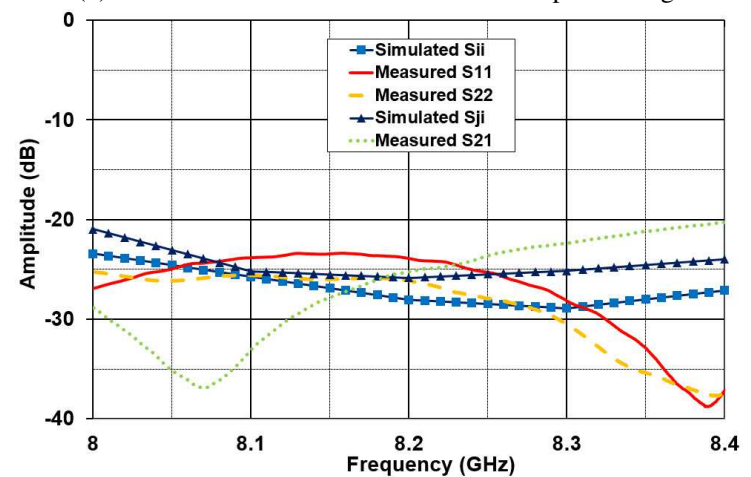

Fig. 7. Comparison of Simulated and measured [S] parameters

2) Measured RHCP radiation patterns

Fig. 8 shows realized RHCP gain radiation patterns drawn in the $\varphi=0^{\circ}$ cut-plane in simulation and measurement (measurements performed by ArianeGroup). The measured directivity and the realized gain are close. It is not possible to give the total losses because of the gain uncertainty measurements of the antenna test range which is $\pm 0.5 \mathrm{~dB}$. An isoflux shape over the entire frequency band is obtained but the EOC angle is at the $\theta=50^{\circ}$ angle instead of $\theta=65^{\circ}$ whatever the frequency. The high value of the P.E.E.K dielectric constant and a mechanically feasible cone prevent this specification. However, this angle is considered sufficient for the intended applications.

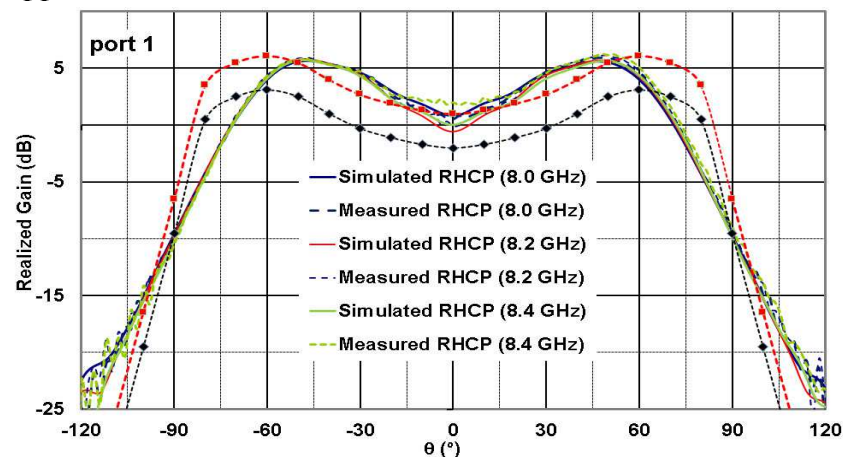

Fig. 8. Comparison of Simulated and measured radiation patterns $\left(\varphi=0^{\circ}\right)$ 
Moreover, this antenna offers strong advantages such as high stability of the boresight gains versus frequency $( \pm 0.7 \mathrm{~dB}$ in simulation and $\pm 1.0 \mathrm{~dB}$ in measurement) for the benefit of the mission link budget. The amplitude roll-off from antenna boresight to horizon is about $10 \mathrm{~dB}$ for low sensitivity with respect to the platform as shown only in the example in Fig. 9 on the realized and AR radiation patterns in the worst case $(\varphi=$ $\left.0^{\circ}\right)$. Simulations on platform were done by ArianeGroup.

Fig. 10 shows the simulated and measured RHCP radiation patterns $(8.2 \mathrm{GHz})$, obtained in different azimuthal cut planes which are quite similar. The radiation patterns are symmetrical as expected. The results are the same for the other port (port 2) except the polarization sense (LHCP).
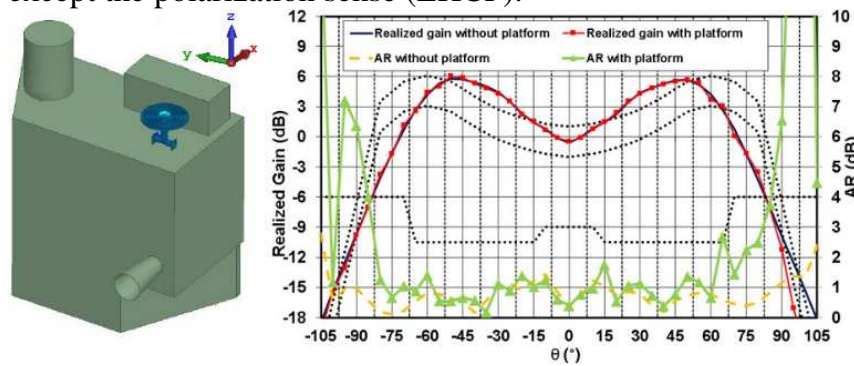

Fig. 9. Comparison of simulated realized and AR radiation patterns $\left(\varphi=0^{\circ}\right)$ with and without platform.

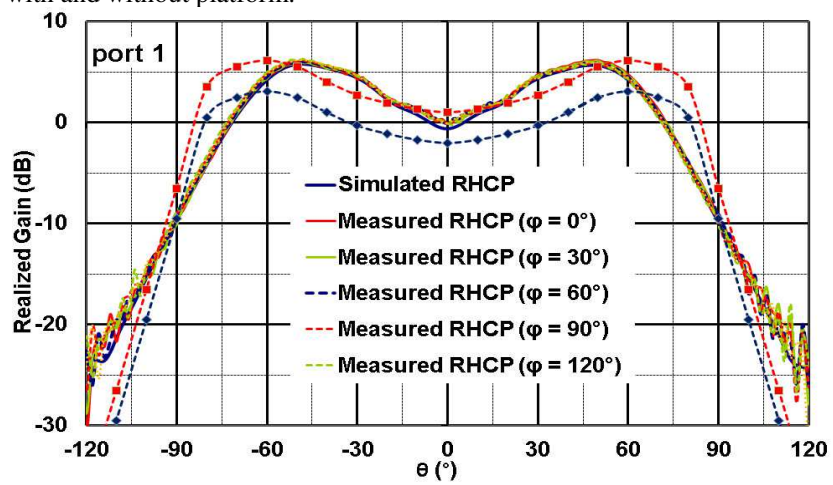

Fig. 10. Simulated and measured RG rad. patterns for different $\varphi$ cut-planes

3) Axial Ratio Radiation pattern

Another strong advantage of this design is the simultaneous dual circular polarization capability due to a very low axial ratio. Fig. 11 and Fig. 12 present the simulated and measured AR for the port 1 . The same results are obtained for the port 2 .

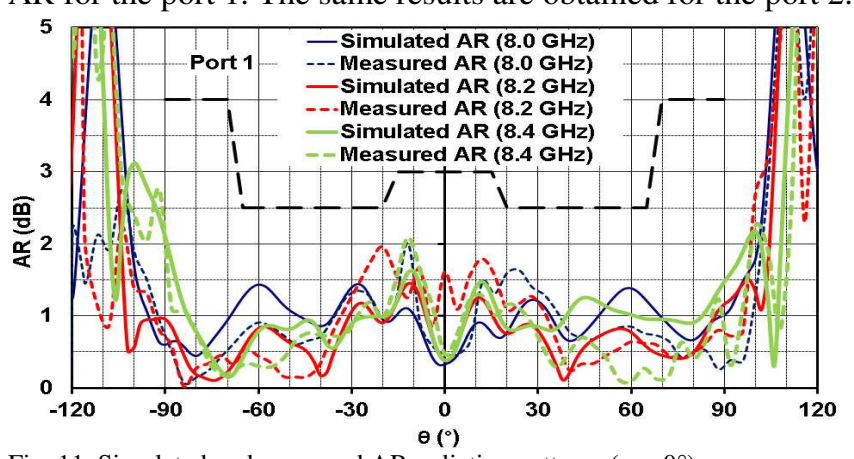

Fig. 11. Simulated and measured AR radiation patterns $\left(\varphi=0^{\circ}\right)$

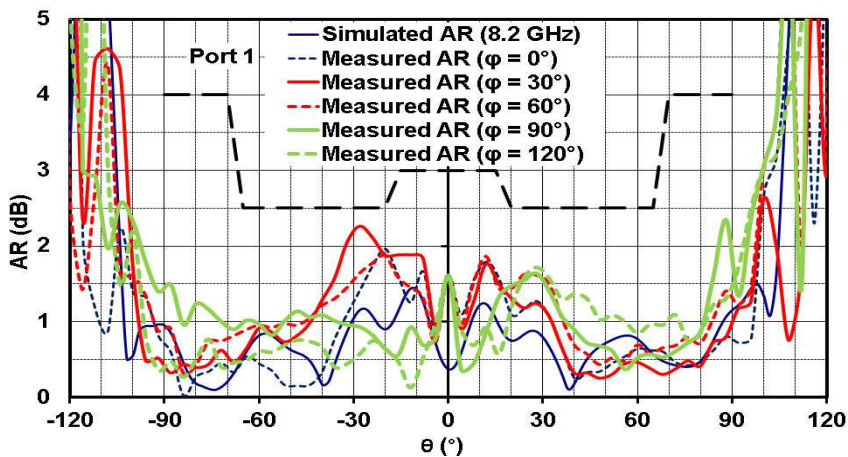

Fig. 12. Simulated and measured AR radiation patterns for $\varphi$ cut-planes

\section{CONCLUSION}

This paper presents a compact isoflux X-band payload telemetry and data handling antenna with simultaneous dual circular polarization for LEO Satellite Applications and materials for space missions. It combines good electromagnetic performances, compactness and low weight validated by experimental results, especially on axial ratio and realized gain.

\section{REFERENCES}

[1] Kilgus, C.C., "Shaped-conical radiation pattern performance of the backfire quadrifilar helix," Antennas and Propagation, IEEE Transactions on, vol.23, no.3, pp.392,397, May 1975. doi: 10.1109/TAP.1975.1141084

[2] https://www.ruag.com/sites/default/files/media_document/2018-03/Xband\%20Data\%20Downlink\%20Antennas_\%20january\%202018_final. pdf

[3] Milligan, T.A.: Modern Antenna Design, 2nd ed., Wiley-IEEE Press, Published by John Wiley \& Sons, Inc., Hoboken, New Jersey, 2005. ISBN: 978-0-471-45776-3.

[4] GEYER, H.: 'Runder Hornstrahler mit ringformigen Sperrtopfen zur gleichzeitigen Ubertragung zweier polarisationsentkoppelter Wellen', Frequenz, 1966, 20, pp. 22-28(especially p. 27)

[5] A. LaGrone and G. Roberts, "Minor lobe suppression in a rectangular horn antenna through the utilization of a high impedance choke flange," in IEEE Transactions on Antennas and Propagation, vol. 14, no. 1, pp. 102-104, January 1966. doi: 10.1109/TAP.1966.1138627

[6] R. Wohlleben, H. Mattes and O. Lochner, "Simple small primary feed for large opening angles and high aperture efficiency," in Electronics Letters, vol. 8, no. 19, pp. 474-476, 21 September 1972. doi: 10.1049/el:19720341

[7] L. Shafai, "Broadening of primary feed patterns by small E-plane slots," in Electronics Letters, vol. 13, no. 4, pp. 102-103, 17 February 1977.

[8] P. Brachat, "Sectoral pattern synthesis with primary feeds," in IEEE Transactions on Antennas and Propagation, vol. 42, no. 4, pp. 484-491, April 1994. doi: 10.1109/8.286216

[9] Kyung-Jin Jeon; Kyo-il Lee; Jae-gi Son; Taek-Kyung Lee; Lee, J. -W; Woo-Kyung Lee, "X-band isoflux pattern antenna for SAR data transmission," Synthetic Aperture Radar (APSAR), 2011 3rd Int. AsiaPacific Conference on, pp.1,4, 26-30 Sept. 2011

[10] J. H. Kim, J. G. Son, S. S. Yoon, J. W. Lee, Taek Kyung Lee and W. K. Lee, "Novel waveguide transition structure for Isoflux antenna in satellite communication," 201215 International Symposium on Antenna Technology and Applied Electromagnetics, Toulouse, 2012, pp. 1-4.

[11] Ravanelli, R.; Iannicelli, C.; Baldecchi, N.; Franchini, F., "Multiobjective optimization of an isoflux antenna for LEO satellite downhandling link," Microwave Radar and Wireless Communications (MIKON), 2010 18th Int.Conference on, pp.1,4, 14-16 June 2010

[12] M. Kilian, C. Hartwanger, A. Schinagl-Weiß and M. Schneider, "X-band downlink antenna characterised by isoflux gain mask," 2018 11th German Microwave Conference (GeMiC), Freiburg, 2018, pp. 21-24. 
[13] F. Caminita et al., "A New Double Polarization Isoflux Antenna," 2019 13th European Conference on Antennas and Propagation (EuCAP), Krakow, Poland, 2019, pp. 1-3.

[14] Antenne pour satellite spatial by E. Arnaud, J. Dugenet, A. Girardot, D. Guihard, T. Monédière, M. Thévenot. (2018, August 18). Patent FR 1857700

[15] D. Davis, O. Digiondomenico, J. Kempic., "A new type of circularly polarized antenna element", Antennas and Propagation Society International Symposium, Oct 1967

[16] E. G. A. Goodall, "Hemi-isotropic radiators for the S- or X-band," in Proceedings of the IEE - Part B: Electronic and Communication Engineering, vol. 106, no. 27, pp. 318-320, May 1959. 